\title{
Preface Issue 1-2014
}

\section{Hans-Christoph Grunau}

(C) Deutsche Mathematiker-Vereinigung and Springer-Verlag Berlin Heidelberg 2014

Last year, the Zentralblatt für Mathematik and the Jahresbericht der DMV began a collaboration to review again classical books and papers and the effect that they have had or could have had on the development of specific mathematical branches from today's point of view. It has turned out that these renewed reviews build a category of articles of its own and that they cannot in general be considered as book reviews or historical articles. For this reason we introduce the new category "Classics Revisited" and shall try to have one article of this kind in each issue. We begin with a new review by Jean-Pierre Otal of William Thurston's 1982 paper on "Three-dimensional manifolds, Kleinian groups and hyperbolic geometry". This research announcement did not only contain his Geometrization Conjecture and his Hyperbolization Theorem for so called Haken manifolds but also a list of 24 of at that time open problems, of which 22 had been solved by 2012. Only few papers have been of such a strong influence and inspiration.

Bosons are particles in quantum mechanics which do not obey the Pauli principle. If a large fraction of particles in a Bose fluid occupies the same quantum state, the so called Bose-Einstein condensation occurs and leads to physically remarkable phenomena like superfluidity. This means that the viscosity of a fluid vanishes completely at low temperatures. Robert Seiringer's survey article on "The excitation spectrum for Bose fluids with weak interaction" reviews recent progress in understanding underlying mathematical models.

Mass aggregation phenomena like e.g. polymerisation may be modelled by means of Smoluchowski's coagulation equation. Barbara Niethammer's survey article addresses the key question in understanding the large time behaviour of solutions

\footnotetext{
H.-Ch. Grunau ( $ه)$

Institut für Analysis und Numerik, Fakultät für Mathematik, Otto-von-Guericke-Universität, Postfach 4120, 39016 Magdeburg, Germany

e-mail: hans-christoph.grunau@ovgu.de
} 
whether this can be described with the help of particular self-similar solutions. Existence and uniqueness results are reviewed and some recent and more elegant proofs are indicated.

Last but not least, recently released books concerning "Clifford algebras and Lie theory", "Mathematics in image processing", and "Oblique derivative problems for elliptic equations" are reviewed. 